\title{
Interleukin 6 Plasma Concentration Associates with Cognitive Decline: The Northern Manhattan Study
}

\author{
Alexis Economos ${ }^{a, b}$ Clinton B. Wright ${ }^{a-c}$ Yeseon Park Moon ${ }^{d}$ Tatjana Rundek ${ }^{a-c}$ \\ LeRoy Rabbani $^{\mathrm{e}}$ Myunghee C. Paik ${ }^{f}$ Ralph L. Sacco ${ }^{\mathrm{a}-\mathrm{c}}$ Mitchell S.V. Elkind $^{\mathrm{c} g}$ \\ ${ }^{a}$ Evelyn F. McKnight Brain Institute, Departments of ${ }^{b}$ Neurology and ${ }^{c}$ Epidemiology and Public Health, \\ Leonard M. Miller School of Medicine, University of Miami, Miami, Fla., and Departments of ${ }^{\mathrm{N}}$ Neurology and \\ eMedicine, College of Physicians and Surgeons, and Departments of ${ }^{f}$ Biostatistics and ${ }^{9}$ Epidemiology, Mailman \\ School of Public Health, Columbia University, New York, N.Y., USA
}

\section{Key Words}

Cognitive decline $\cdot$ Cohort studies • Interleukin 6 • Inflammation

\begin{abstract}
Background: Interleukin 6 (IL-6) is an inflammatory cytokine that has been associated with vascular disease and cognitive impairment, but few studies have examined these relationships in population-based studies that include Hispanic and Black people who often have a greater prevalence of vascular risk factors and are at an elevated risk of dementia than Whites. We examined relative elevations of plasma IL- 6 concentrations in relation to cognitive decline in a stroke-free racially/ethnically diverse community-based sample from Northern Manhattan. Methods: We used mixed effects models to measure the effect of IL- 6 on change in performance on the modified Telephone Interview for Cognitive Status (TICS-m) measured annually in our cohort, adjusting for sociodemographic and vascular risk factors. Results: There were 1,224 participants with IL-6 levels (median $1.5 \mathrm{pg} /$ $\mathrm{ml}$, interquartile range $0.83-2.57 \mathrm{pg} / \mathrm{ml}$ ) and TICS-m data available (mean $=31.6$ points, SD 6.5). The mean age was 71 (SD 9.3; 64\% women, 59\% Hispanic, 19\% Black, 19\% White) with 3,406 person-years and a median 3.0 years of follow-up
\end{abstract}

(interquartile range 1.1-4.0 years). Participants with IL-6 levels above the median showed greater cognitive decline on the TICS-m compared to those with levels below the median, adjusting for sociodemographic and vascular factors ( $\beta=$ -0.17 points/year, $p=0.02$ ). Decline on the TICS-m among participants with IL- 6 above the median differed by age ( $p$ for interaction $<0.001$ ). There was no interaction by race/ ethnicity, vascular risk factors, C-reactive protein, apolipoprotein $\varepsilon 4$ allele status, or the metabolic syndrome among nondiabetics. Conclusions: IL- 6 associated with cognitive decline among older participants in this racially/ethnically diverse sample independent of other vascular risk factors and C-reactive protein.

Copyright $\odot 2013$ S. Karger AG, Basel

\section{Introduction}

Interleukin 6 (IL-6) is an inflammatory cytokine linked to dementia, mild cognitive impairment and Alzheimer disease [1-4]. However, there are conflicting data on the effect of IL-6 on cognitive decline. Several studies have reported an association, but the Dutch Longitudinal Aging Study Amsterdam found IL-6 was not associated with cognitive decline, and the Whitehall II Study found

\section{KARGER}

E-Mail karger@karger.com

www.karger.com/ned
C 2013 S. Karger AG, Basel

0251-5350/13/0404-0253\$38.00/0
Clinton Wright, MD, MS

Evelyn F. McKnight Brain Institute

1120 NW 14th Street, CRB-1349

Miami, FL 33136 (USA)

E-Mail cwright@ med.miami.edu 
raised levels of IL-6 had an inconsistent association with poor cognitive performance [5-9].

The role of traditional vascular risk factors in mediating an association between IL- 6 and cognitive decline requires further clarification as well [10]. IL-6 combined with the constellation of risk factors making up the metabolic syndrome may increase the risk of cognitive decline. The Leiden 85-Plus Study found that the combination of cardiovascular disease and a proinflammatory cytokine response may be associated with cognitive impairment $[11,12]$. On the other hand, the Edinburgh Artery Study found that IL- 6 was associated with cognitive decline independent of cardiovascular disease (CVD) [13]. We have previously reported an inverse association between IL- 6 and cognitive performance independent of carotid intima-media thickness, a subclinical marker of CVD [14]. In addition, IL-6 stimulates C-reactive protein (CRP) production, an established marker of cardiovascular risk, but there is conflicting data regarding the association of CRP with cognitive decline in combination with elevated IL-6 [6, 12]. In an African-Caribbean population, IL-6 was associated with cognitive decline, even when adjusted for vascular risk factors; however, CRP was not found to exhibit such an association [15].

Prospective studies examining the relationship between IL- 6 and cognitive function have found conflicting results, and it is unclear if vascular or neurodegenerative processes underlie the associations [5, 7, 9, 11, 13-15]. In addition, few studies on IL- 6 and cognitive decline have included Hispanics and non-Hispanic Blacks who may be at greater risk of dementia than non-Hispanic Whites [10, $16,17]$. We conducted a prospective study of the association between baseline plasma IL- 6 levels and cognitive decline, and examined vascular risk factors, apolipoprotein $\varepsilon 4$ (APOE4) allele status, and inflammation in a stroke-free community-based multiethnic cohort.

\section{Methods}

\section{Description of Cohort}

The Northern Manhattan Study (NOMAS) includes a prospective cohort of 3,298 stroke-free participants recruited using random digit dialing in northern Manhattan between 1993 and 2001. The details of enrollment are described elsewhere [18]. Briefly, participants were eligible if they met the following conditions: (1) no history of stroke, (2) age $\geq 40$ years and (3) residence in a household with a telephone for at least 3 months in northern Manhattan. Written informed consent was obtained from all participants, and the Columbia University Medical Center and University of Miami Miller School of Medicine Institutional Review Boards approved the study.

\section{Baseline Evaluation and Follow-Up}

Trained bilingual research assistants and study physicians collected demographic, medical and laboratory data at enrollment using standardized data collection techniques and risk factor questions. These standardized questions were adapted from the Behavioral Risk Factor Surveillance System regarding the following conditions: hypertension, diabetes mellitus and cigarette smoking $[19,20]$. Depressive symptoms were quantified using the Hamilton Depression Scale [21]. Subjects were contacted annually via telephone starting in 1998 to document information regarding illnesses, hospitalizations, vital status and cardiovascular events.

\section{Laboratory Measurements}

Serum samples for IL-6 and high-sensitivity CRP (hsCRP) were drawn into EDTA tubes at baseline, spun immediately at $3,000 \mathrm{~g}$ at $4^{\circ} \mathrm{C}$ for $20 \mathrm{~min}$, and then frozen at $-70^{\circ} \mathrm{C}$ for later analysis. Inflammatory marker levels were then measured in batched samples by enzyme-linked immunosorbent assay using monoclonal antibodies to IL- 6 with a lower limit of detection of $0.1 \mathrm{pg} /$ $\mathrm{ml}$ (Biosource International, Camarillo, Calif., USA) and hsCRP with a lower limit of detection of $0.1 \mathrm{mg} / 1$ (BioCheck Inc., Foster City, Calif., USA). ApoE $\varepsilon 2, \varepsilon 3$ and $\varepsilon 4$ alleles were determined by two single-nucleotide polymorphisms, i.e. $r s 429358$ and $r s 7412$. The two single-nucleotide polymorphisms were genotyped using $\operatorname{TaqMan}^{\circledR}$ allelic discrimination assays (Applied Biosystems) following the manufacturer's instructions.

\section{Cognitive Assessment}

Cognitive status was estimated by trained research assistants using the modified Telephone Interview for Cognitive Status (TICS-m), and has been included in the annual follow-up since 2001. The original TICS was based on the Mini Mental State Examination and was designed to assess global cognition by testing the following domains: attention, language, calculation, and immediate recall of 10 words. The modified TICS-m includes recall of the 10-word list after a short delay, and has been validated in clinical and research settings $[22,23]$. Details of the study cohort with both IL- 6 and TICS-m measurements may be found in figure 1 and the number of participants with different numbers of TICS-m evaluations is shown in table 1.

\section{Covariates}

Established risk factors for cognitive impairment were selected as covariates for multivariable analysis. All variables were assessed at baseline. Race/ethnicity was based on self-identification. Educational status was dichotomized based on whether or not high school had been completed. Insurance status was dichotomized as Medicaid or no insurance versus Medicare or private insurance. A history of depression was defined as a Hamilton Depression Scale score greater than 10 or current antidepressant use. Moderate physical activity was dichotomized as any recreational or physical activity in the prior 2 weeks versus none, according to our validated methods [20]. Diabetes was defined based on the subject's self-reported history, usage of hypoglycemic medications, or fasting blood sugar greater than $126 \mathrm{mg} / \mathrm{dl}[19,20]$. Smoking status was categorized as never, current (within the last year) or former smoker. Reported alcohol consumption was categorized as moderate ( $1 \mathrm{drink} /$ week to 2 drinks/day) versus light $(<1$ drink/week) or nondrinkers. The number of heavy drinkers 
Fig. 1. Flow diagram of participant inclu-

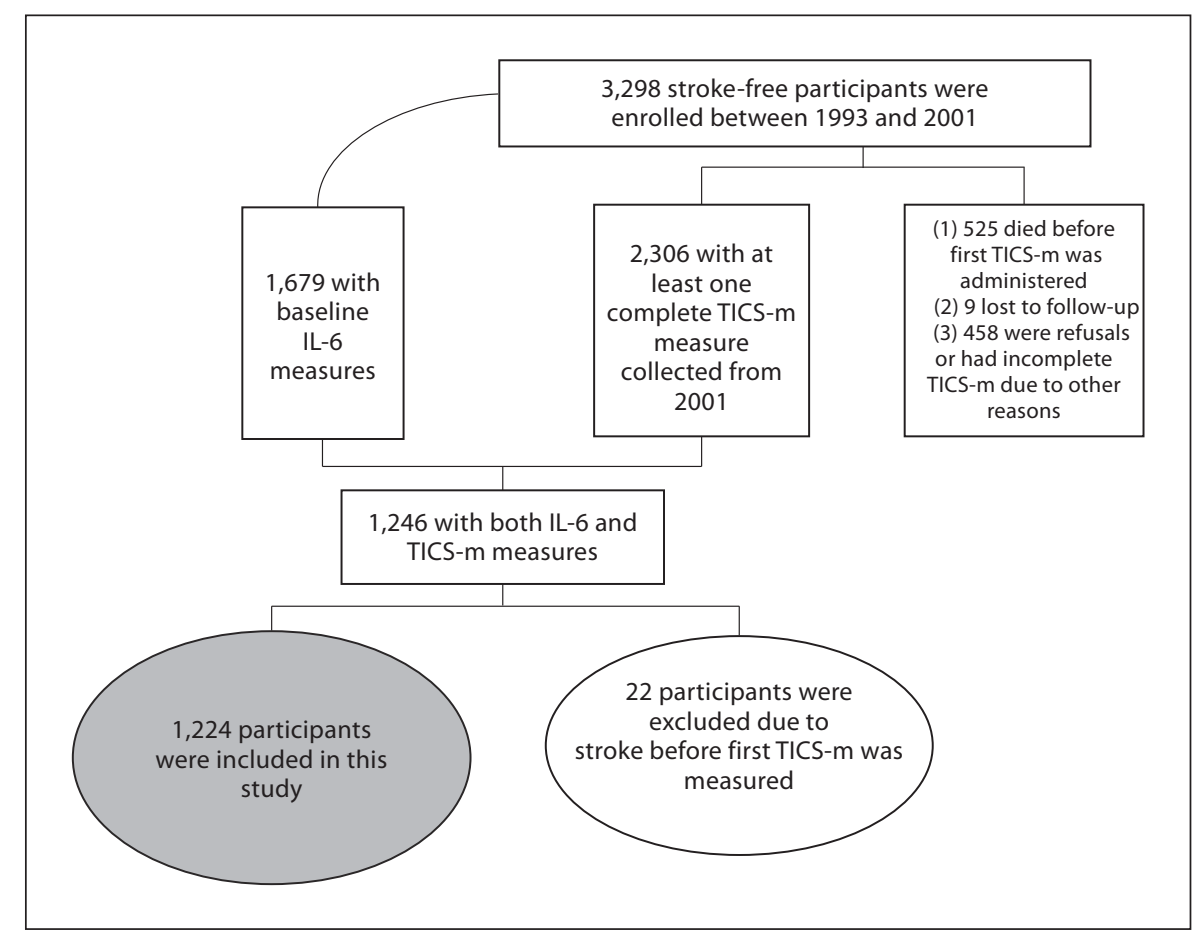

Table 1. Number and percentage (in parentheses) of participants with different numbers of TICS-m assessments

\begin{tabular}{ll}
\hline Number of TICS-m & Patients \\
\hline 1 & $201(16)$ \\
2 & $213(17)$ \\
3 & $273(22)$ \\
4 & $256(21)$ \\
$5+$ & $281(24)$ \\
\hline
\end{tabular}

( $>2$ drinks/day) was small and was included in the reference category. Metabolic syndrome was defined by ATP III criteria as three of the following: abdominal obesity, raised triglycerides, reduced high-density lipoprotein cholesterol, elevated blood pressure, and raised plasma glucose [24].

\section{Statistical Analyses}

We compared sample characteristics across levels of IL-6 using $\chi^{2}$ and Student $t$ tests as appropriate. We used mixed effects models with random intercepts to examine the association between IL-6 levels and cognitive decline measured with the TICS$\mathrm{m}$. There was no graded dose effect across quartiles, but the effect differed at the median split. We therefore created a categorical variable for IL- 6 levels above and below the median. To estimate the effect of IL- 6 and other covariates on change in TICS-m score over time, we examined the interaction between IL- 6 and time between baseline and subsequent TICS-m measurement. The resulting coefficient provides the slope of change of TICS-m scores over time and is a standard approach [25]. In addition to IL-6, we examined the effect of hsCRP on change in TICS-m scores over time as a continuous variable, using quartiles of hsCRP, and using the established cutoffs of $>3,1-3$ and $<1 \mathrm{mg} / \mathrm{dl}$. To relate the rate of cognitive decline associated with these biomarkers to age-related change in TICS-m scores in our sample, we also examined age as a predictor of cognitive decline. We looked for effect modification by examining interactions between IL- 6 , time and other covariates of interest such as age and CRP. Finally, we carried out two subanalyses, one to examine an interaction with metabolic syndrome among participants without diabetes and the other to examine the effect of APOE4 allele status. To allow us to estimate the effect of IL- 6 on cognitive decline in a stroke-free population, for the primary analysis participants with stroke prior to the baseline TICS-m were excluded and we censored TICS-m scores acquired after a stroke event.

\section{Results}

There were 1,224 NOMAS participants with both IL-6 (median $1.5 \mathrm{pg} / \mathrm{ml}$, interquartile range $0.83-2.57 \mathrm{pg} / \mathrm{ml}$ ) and TICS-m measurements (overall mean $=31.6$ points, $\mathrm{SD}=6.5$ ). Baseline characteristics of the sample are shown in table 2. The interquartile range of scores for the first TICS-m done on participants was 26-35. Compared 
Table 2. Baseline characteristics (number with percentage in parentheses) of the sample $(n=1,224)$ with IL- 6 and TICS-m measurements

\begin{tabular}{|c|c|c|c|c|}
\hline \multirow[t]{2}{*}{ Characteristic } & \multicolumn{3}{|l|}{ IL-6 } & \multirow[t]{2}{*}{$\mathrm{p}$ value } \\
\hline & $<$ median & $>$ median & total & \\
\hline \multicolumn{5}{|l|}{ Sex } \\
\hline Men & $237(54)$ & $204(46)$ & 441 & \multirow[t]{2}{*}{0.0496} \\
\hline Women & $375(48)$ & $408(52)$ & 783 & \\
\hline \multicolumn{5}{|l|}{ Race/ethnicity } \\
\hline White & $125(53)$ & $109(47)$ & 234 & ref. \\
\hline Black & $116(50)$ & $115(50)$ & 231 & 0.216 \\
\hline Other & $18(51)$ & $17(49)$ & 35 & n.a. \\
\hline Hispanic & $353(49)$ & $371(51)$ & 724 & 0.49 \\
\hline \multicolumn{5}{|l|}{ Education } \\
\hline$<$ High school & $305(47)$ & $342(53)$ & 647 & \multirow[t]{2}{*}{0.035} \\
\hline$\geq$ High school & $307(53)$ & $270(47)$ & 577 & \\
\hline \multicolumn{5}{|l|}{ Medical insurance } \\
\hline Medicare or private & $344(53)$ & $306(47)$ & 650 & \multirow[t]{2}{*}{0.025} \\
\hline Medicaid or none & $266(46)$ & $306(54)$ & 572 & \\
\hline \multicolumn{5}{|l|}{ Current smoker } \\
\hline No & $537(52)$ & $492(48)$ & 1,029 & \multirow[t]{2}{*}{0.0005} \\
\hline Yes & $75(38)$ & $120(62)$ & 195 & \\
\hline \multicolumn{5}{|l|}{ Diabetes } \\
\hline No & $530(50)$ & $524(50)$ & 1,054 & \multirow[t]{2}{*}{0.669} \\
\hline Yes & $81(49)$ & $86(51)$ & 167 & \\
\hline \multicolumn{5}{|c|}{ Moderate alcohol consumption } \\
\hline No & $365(48)$ & $398(52)$ & 763 & \multirow[t]{2}{*}{0.047} \\
\hline Yes & $246(54)$ & $212(46)$ & 458 & \\
\hline \multicolumn{5}{|l|}{ Hypertension } \\
\hline No & $198(57)$ & $152(43)$ & 350 & \multirow[t]{2}{*}{0.0037} \\
\hline Yes & $414(47)$ & $460(53)$ & 874 & \\
\hline \multicolumn{5}{|l|}{ Any physical activity } \\
\hline No & $262(49)$ & $268(51)$ & 530 & \multirow[t]{2}{*}{0.729} \\
\hline Yes & $350(50)$ & $344(50)$ & 694 & \\
\hline \multicolumn{5}{|l|}{ Depression } \\
\hline No & $558(51)$ & $541(49)$ & 1,099 & \multirow[t]{2}{*}{0.1045} \\
\hline Yes & $52(43)$ & $69(57)$ & 121 & \\
\hline \multicolumn{5}{|l|}{ Metabolic syndrome } \\
\hline No & $409(57)$ & $308(43)$ & 717 & \multirow[t]{2}{*}{$<0.0001$} \\
\hline Yes & $203(40)$ & $304(60)$ & 507 & \\
\hline
\end{tabular}

Diabetes was defined as a fasting blood glucose of $>126 \mathrm{mg} / \mathrm{dl}$ or a history of insulin or hypoglycemic use. Moderate alcohol consumption was defined as taking more than 1 alcoholic beverage per month up to 2 per day. Depression was defined as a Hamilton Depression Scale score $>10$ or current antidepressant use.

to NOMAS participants without these measurements, the current sample included a greater proportion of Hispanics (59 vs. $48 \%$ ), more participants with a high school education (47 vs. $45 \%$ ) and fewer people with diabetes (14 vs. $27 \%)$. Twenty-two participants were excluded due to
Table 3. Multivariable analysis of IL- 6 as a predictor of change in the TICS-m score

\begin{tabular}{lll}
\hline Dichotomized IL-6 & Parameter estimate & $\mathrm{p}$ \\
\hline $\begin{array}{l}\text { Unadjusted } \\
\text { IL-6 above mean }\end{array}$ & $\begin{array}{l}-0.19(-0.32,-0.06) \\
\text { reference }\end{array}$ & 0.006 \\
IL-6 below mean & $\begin{array}{l}\text { Model 1 } \\
\text { IL-6 above mean }\end{array}$ & $\begin{array}{l}-0.17(-0.30,-0.36) \\
\text { reference }\end{array}$ \\
IL-6 below mean & 0.013 \\
Model 2 & \\
IL-6 above mean & $\begin{array}{l}-0.17(-0.30,-0.03) \\
\text { IL-6 below mean }\end{array}$ & 0.015 \\
\hline
\end{tabular}

Parameter estimate represents the average point decline per year in the TICS-m score; figures in parentheses indicate $95 \%$ confidence intervals. Model 1: adjusted for age, race/ethnicity, education, gender, and insurance status, depression, physical activity. Model 2: adjusted for covariates in model 1 plus diabetes mellitus, alcohol consumption and smoking status.

stroke prior to baseline TICS-m assessment, and $13 \mathrm{ob}-$ servations were censored after stroke.

Compared to those with IL-6 levels below the median, participants with IL-6 levels above the median were older, more likely to have hypertension, the metabolic syndrome, to be women, to have less than a high school education, Medicaid or no insurance, to be current smokers, and to report moderate alcohol consumption (table 2). Over 3,406 person-years of follow-up (median 3.0 years, interquartile range 1.1-4.0; see table 1 for number of participants with different numbers of TICS-m measurements), we found that participants with IL-6 levels above the median had a greater decline on the TICS-m over time than those with levels below the median (table 3). This effect was slightly attenuated after adjusting for age, race/ethnicity, education, sex and insurance status but remained significant. The strength of the association between IL- 6 and cognitive decline remained unchanged after adjusting for diabetes mellitus, hypertension, physical activity, depression, alcohol consumption and smoking status (table 3).

We found a significant interaction between IL-6, decline on TICS-m over time, and age $(\beta=-0.40$ points/ year for each additional year of age above the sample mean in those with IL- 6 levels above the median compared to below, $\mathrm{p}$ for 3 -way interaction $<0.0001)$. Further stratifying by age, participants older than 71 with IL- 6 levels above the median declined by 0.4 points more per year than those with IL- 6 levels below the median $(\mathrm{p}<$ 
0.0001), while in younger participants IL-6 levels were not significantly associated with the rate of cognitive decline. We found no interaction by race/ethnicity or by modifiable vascular risk factors. Our findings were confirmed among a subsample limited to nondiabetics ( $\mathrm{n}=$ 1,054): those with IL-6 levels above the median still showed a greater rate of decline on the TICS- $m$ than below the median $(\beta=-0.17$ points/year, $p=0.02)$, and there was no interaction with metabolic syndrome (data not shown). We did not examine the association between IL- 6 and change in TICS-m scores among diabetics due to the small sample size $(\mathrm{n}=167)$.

hsCRP (median $2.5 \mathrm{mg} / \mathrm{l}$, interquartile range 1.0-5.7 $\mathrm{mg} / \mathrm{l}$ ) was not associated with cognitive decline on the TICS-m. In addition, change in TICS-m scores did not differ significantly for those with hsCRP levels in any of the upper three quartiles compared to those with hsCRP levels in the lowest quartile $(\mathrm{Q} 4 \beta=-0.01$ points/year; $\mathrm{Q} 3$ $\beta=-0.001$; 2 2 $\beta=0.05$ each compared to Q1, $p>0.1$ for all comparisons) or for those with hsCRP levels $>3 \mathrm{mg} / \mathrm{l}$ ( $\beta=-0.05, p=0.50)$, or hsCRP levels between 1 and 3 $\mathrm{mg} / \mathrm{l}(\beta=0.02, \mathrm{p}=0.83)$, compared to those below $1 \mathrm{mg} / \mathrm{l}$. There was also no interaction between IL- 6 and hsCRP in relation to cognitive decline (data not shown).

Examining APOE4 allele carrier status as a possible moderator of the association between IL- 6 and TICS-m performance over time among a subgroup for whom allele status was available $(\mathrm{n}=817)$, we included APOE4 as a covariate in our fully adjusted model and the association between IL- 6 and cognitive decline diminished and was no longer significant $(\beta=-0.13, p=0.1)$. There was no significant interaction, however, and thus the greater decline in TICS-m scores seen for those with IL-6 levels above the median did not differ by APOE4 status (data not shown).

Finally, to put our findings regarding IL- 6 and cognitive decline into perspective, we examined the effect of age on change in TICS-m scores over time. Participant age was associated with a decline of 0.02 points/year $(\mathrm{p}<$ $0.001)$.

\section{Discussion}

Participants with baseline serum IL- 6 values above the median had greater cognitive decline than those below the median in this prospective stroke-free racially/ethnically diverse urban US population. The magnitude of decline in TICS-m scores among those with IL-6 levels above the median compared to below the median was

IL-6 Plasma Concentration Associates with Cognitive Decline equivalent to the average amount of cognitive aging occurring in this sample over about 8 years. Based on a stratified analysis, the effect of IL- 6 on cognitive decline was significant only among those older than the mean age of 71 , independently of other sociodemographic and vascular risk factors.

The role of IL- 6 in cognitive decline is poorly understood, but an association between relative elevations of IL-6 and CVD, as well as CVD risk factors, has been found in prior studies. For example, serum IL-6 levels have been associated with a greater risk of recurrent stroke, as well as greater stroke severity, than lower levels of the marker, but causality in either case has not been determined [26]. The Health, Aging, and Body Composition (Health ABC) study showed that those with the metabolic syndrome were at greater risk of cognitive decline than those without the metabolic syndrome only if they had both CRP and IL- 6 levels above the median [11]. In the current study, the association between IL-6 and cognitive decline did not depend on having metabolic syndrome even when restricted to nondiabetics. In addition, hsCRP was not a predictor of cognitive decline in this sample, and there was no interaction between relative elevations of IL- 6 and hsCRP and cognitive decline.

We also found that other vascular risk factors did not alter the strength of the association between IL- 6 and cognitive decline. The populations in NOMAS and Health $\mathrm{ABC}$ are distinct, and we were unable to find studies of the association between IL- 6 and cognitive decline that have included large samples of Hispanic people, while the Health ABC study included well-functioning White and African-American elders. In NOMAS the risk of cognitive decline for those with IL-6 levels above the median did not differ by racial/ethnic group. While hypertension is very common among all three racial/ ethnic groups in NOMAS, as in Health $\mathrm{ABC}$, there are some differences as well. For example, high triglycerides and low high-density lipoprotein cholesterol were common among Hispanics but less so for both Whites and Blacks in our sample. Some of these differences may explain disparate findings between these studies regarding the role of IL- 6 and cognitive decline. hsCRP is an established inflammatory marker for future vascular disease; however, data on hsCRP in the Hispanic population is scarce. The NOMAS population, of which approximately $52 \%$ are Hispanic, had higher hsCRP levels than whites [27].

It is not clear if IL-6 is playing a role in cognitive dysfunction through vascular damage. A genetic propensity 
to a heightened inflammatory response predisposing to cognitive impairment has been proposed, however, and some of these studies support a vascular mechanism [11]. We have previously reported that individuals homozygous for the GG genotype at position 174 of the IL- 6 gene had greater carotid artery wall thickness, and the Atherosclerosis Risk in Communities Study showed an association between cognitive dysfunction and increased carotid intima-media thickness $[28,29]$. However, adjusting for baseline vascular risk factors did not alter the association between IL- 6 and cognitive decline in our study, suggesting that a nonvascular mechanism may be operative. Another plausible mechanism by which IL- 6 could influence the rate of cognitive decline is through a neurodegenerative process, and one study in a community sample of middle-aged adults has found that elevated plasma IL-6 levels were associated with lower hippocampal gray matter volumes independently of body fat and hypertension [30]. In the current study, adjusting for APOE4 allele status in a subsample attenuated the effect of IL- 6 on cognitive decline, and it was no longer significant, suggesting possible mediation. However, there was no significant interaction between IL-6, change in TICS-m scores over time, and APOE4 status. It is likely that our power to detect both the main effect of IL- 6 on cognitive decline and the interaction between IL- 6 and APOE4 status was limited by the smaller sample size. Larger studies are needed to clarify this finding. Interestingly, the Rotterdam study, contrary to what was hypothesized, found that the risk for dementia with increased levels of IL- 6 was independent of atherosclerosis, and the authors proposed a peripheral inflammatory mechanism related to $\beta$-amyloid deposition [31].

An important limitation of this study is that there are limited data showing that IL- 6 crosses the blood-brain barrier, and that serum IL-6 may accurately reflect cerebrospinal fluid levels [32]. Another concern is that serum IL-6 levels were measured only at baseline in NOMAS, and thus it is possible that levels changed later. However, studies examining the validity of IL- 6 levels show that intraindividual concentrations do not vary much over time, though the time periods examined have been short, and therefore remain an indicator of chronic inflammatory states that both precede and follow the actual measurement [33-35]. Another concern is that the TICS-m is a global test of cognition and lacks sensitivity for specific domains, such as verbal memory or executive function. However, this would tend to decrease our ability to detect an association between IL- 6 and cognition. Further data examining more sensitive neuropsychological tests are warranted. Despite the relatively large sample size, the median follow-up per individual was 3 years and studies with a longer follow-up are needed to confirm our findings. In addition, the study sample lacked enough people with diabetes to assess IL-6 levels and cognitive decline in this group. Despite the limitations, this study also has important strengths, including the large sample size, the population-based multiethnic cohort and the longitudinal design.

\section{Conclusion}

In summary, we found that participants in a population-based urban US sample with IL-6 levels above the median had a greater rate of cognitive decline than those with levels below the median. This association was independent of traditional vascular risk factors, and was more pronounced with advancing age. We did not find racial/ ethnic differences in the effect of IL-6 on cognitive decline, and these results are applicable to a racially/ethnically diverse population.

\section{Acknowledgements}

This work is supported by grants from the National Institute of Neurological Disorders and Stroke (R37 NS 29993, K02 NS 059729, K23 42912), the American Heart Association (0735387N), and the Evelyn F. McKnight Brain Institute. The authors would like to thank the staff of the Northern Manhattan Study and especially Janet DeRosa, project manager.

\section{Disclosure Statement}

The authors report no conflicts of interest regarding this paper.

References 
-4 Wright CB, Elkind MSV, Rundek T, BodenAlbala B, Paik MC, Sacco RL: Alcohol intake, carotid plaque, and cognition: the Northern Manhattan Study. Stroke 2006;37:11601164.

5 Schram MT, Euser SM, de Craen AJ, Witteman JC, Frolich M, Hofman A, Jolles J, Breteler MM, Westendorp RG: Systemic markers of inflammation and cognitive decline in old age. J Am Geriatr Soc 2007;55:708-716.

-6 Yaffe K, Lindquist K, Penninx BW, Simonsick EM, Pahor M, Kritchevsky S, Launer L, Kuller L, Rubin S, Harris T: Inflammatory markers and cognition in well-functioning African-American and white elders. Neurology 2003;61:76-80.

7 Weaver JD, Huang MH, Albert M, Harris T, Rowe JW, Seeman TE: Interleukin- 6 and risk of cognitive decline: MacArthur studies of successful aging. Neurology 2002;59:371378.

-8 Gimeno D, Marmot MG, Singh-Manoux A: Inflammatory markers and cognitive function in middle-aged adults: the Whitehall II Study. Psychoneuroendocrinology 2008;33: 1322-1334

-9 Dik MG, Jonker C, Hack CE, Smit JH, Comijs HC, Eikelenboom P: Serum inflammatory proteins and cognitive decline in older persons. Neurology 2005;64:1371-1377.

-10 Patterson CC, Smith AE, Yarnell JW, Rumley A, Ben-Shlomo Y, Lowe GD: The associations of interleukin-6 (IL-6) and downstream inflammatory markers with risk of cardiovascular disease: the Caerphilly Study. Atherosclerosis 2010;209:551-557.

-11 Yaffe K, Kanaya A, Lindquist K, Simonsick EM, Harris T, Shorr RI, Tylavsky FA, Newman $A B$ : The metabolic syndrome, inflammation, and risk of cognitive decline. JAMA 2004;292:2237-2242.

- 12 Van Exel E, de Craen AJM, Remarque EJ, Gussekloo J, Houx P, Bootsma-van der Wiel A, Frolich M, Macfarlane PW, Blauw GJ, Westendorp RGJ: Interaction of atherosclerosis and inflammation in elderly subjects with poor cognitive function. Neurology 2003;61:1695-1701.

13 Rafnsson SB, Deary IJ, Smith FB, Whiteman MC, Rumley A, Lowe GD, Fowkes FG: Cognitive decline and markers of inflammation and hemostasis: the Edinburgh Artery Study. J Am Geriatr Soc 2007;55:700-707.
14 Wright CB, Sacco RL, Rundek T, Delman JB, Rabbani LE, Elkind MSV: Interleukin-6 is associated with cognitive function: the Northern Manhattan Study. J Stroke Cerebrovasc Dis 2006;15:34-38.

15 Jordanova V, Stewart R, Davies E, Sherwood $\mathrm{R}$, Prince M: Markers of inflammation and cognitive decline in an African-Caribbean population. Int J Geriatr Psychiatry 2007;22: 966-973.

16 Gurland BJ, Wilder DE, Lantigua R, Stern Y, Chen J, Killeffer EH, Mayeux R: Rates of dementia in three ethnoracial groups. Int J Geriatr Psychiatry 1999;14:481-493.

17 Tang MX, Cross P, Andrews H, Jacobs DM, Small S, Bell K, Merchant C, Lantigua R, Costa R, Stern Y, Mayeux R: Incidence of AD in African-Americans, Caribbean Hispanics, and Caucasians in northern Manhattan. Neurology 2001;56:49-56.

-18 Wright CB, Lee HS, Paik MC, Stabler SP, Allen RH, Sacco RL: Total homocysteine and cognition in a tri-ethnic cohort: the Northern Manhattan Study. Neurology 2004;63: 254-260.

19 Sacco RL, Elkind M, Boden-Albala B, Lin IF, Kargman DE, Hauser WA, Shea S, Paik MC: The protective effect of moderate alcohol consumption on ischemic stroke. JAMA 1999;281:53-60.

20 Sacco RL, Gan R, Boden-Albala B, Lin IF, Kargman DE, Hauser WA, Shea S, Paik MC: Leisure-time physical activity and ischemic stroke risk: the Northern Manhattan Stroke Study. Stroke 1998;29:380-387.

21 Hamilton M: The Hamilton Depression Rating Scale. J Neurol Neurosurg Psychiatry 1960;23:56-62.

22 Brandt J, Spencer M, Folstein MF: The Telephone Interview for Cognitive Status. Neuropsychiatry Neuropsychol Behav Neurol 1988;1:111-117.

23 Khatri M, Nickolas T, Moon YP, Paik MC, Rundek T, Elkind MS, Sacco RL, Wright CB: CKD associates with cognitive decline. J Am Soc Nephrol 2009;20:2427-2432.

24 Executive Summary of the Third Report of the National Cholesterol Education Program (NCEP) Expert Panel on Detection, Evaluation and Treatment of High Blood Cholesterol in Adults (Adult Treatment Panel III). JAMA 2001;285:2486-2497.

25 Jack CR Jr, Vemuri P, Wiste HJ, Weigand SD, Lesnick TG, Lowe V, Kantarci K, Bernstein MA, Senjem ML, Gunter JL, Boeve BF, Trojanowski JQ, Shaw LM, Aisen PS, Weiner MW, Petersen RC, Knopman DS: Shapes of the trajectories of 5 major biomarkers of Alzheimer disease. Arch Neurol 2012, E-pub ahead of print.
26 Tso AR, Merino JG, Warach S: Interleukin-6 174G/C polymorphism and ischemic stroke: a systematic review. Stroke 2007;38:30703075.

27 Elkind MS, Luna JM, Moon YP, Liu KM, Spitalnik SL, Paik MC, Sacco RL: High-sensitivity C-reactive protein predicts mortality but not stroke: the Northern Manhattan Study. Neurology 2009;73:1300-1307.

-28 Cerhan JR, Folsom AR, Mortimer JA, Shahar E, Knopman DS, McGovern PG, Hays MA, Crum LD, Heiss G: Correlates of cognitive function in middle-aged adults. Atherosclerosis Risk in Communities (ARIC) Study Investigators. Gerontology 1998;44:95-105.

-29 Rundek T, Elkind MS, Pittman J, Boden-Albala B, Martin S, Humphries SE, Juo SH, Sacco RL: Carotid intima-media thickness is associated with allelic variants of stromelysin-1, interleukin-6, and hepatic lipase genes: the Northern Manhattan Prospective Cohort Study. Stroke 2002;33:1420-1423.

- 30 Marsland AL, Gianaros PJ, Abramowitch SM, Manuck SB, Hariri AR: Interleukin-6 covaries inversely with hippocampal grey matter volume in middle-aged adults. Biol Psychiatry 2008;64:484-490.

- 31 Engelhart MJ, Geerlings MI, Meijer J, Kiliaan A, Ruitenberg A, van Swieten JC, Stijnen T, Hofman A, Witteman JCM, Breteler MMB: Inflammatory proteins in plasma and the risk of dementia: the Rotterdam Study. Arch Neurol 2004;61:668-672.

32 Banks WA, Kastin AJ, Broadwell RD: Passage of cytokines across the blood-brain barrier. Neuroimmunomodulation 1995;2:241248

33 Picotte M, Campbell CG, Thorland WG: Day-to-day variation in plasma interleukin- 6 concentrations in older adults. Cytokine 2009;47:162-165.

34 Rao KM, Pieper CS, Currie MS, Cohen HJ: Variability of plasma IL- 6 and crosslinked fibrin dimers over time in community dwelling elderly subjects. Am J Clin Pathol 1994; 102:802-805.

35 Gabay C: Interleukin-6 and chronic inflammation. Arthritis Res Ther 2006;8(suppl 2): S3. 